\title{
The Impact of Acute Loss of Weight on Eustachian Tube Function
}

\author{
Gabriela Pascoto ${ }^{1}$ Cassiana Abreu ${ }^{1}$ Maria Laura Silva ${ }^{1} \quad$ Raimar Weber $^{1}$ Shirley Shizue Pignatari ${ }^{1}$ \\ Aldo Stamm ${ }^{1}$ \\ ${ }^{1}$ Department of Otorhinolaryngology, Complexo Hospitalar Edmundo \\ Vasconcelos, São Paulo, SP, Brazil \\ Int Arch Otorhinolaryngol 2014;18:376-379.
}

\begin{abstract}
Introduction The eustachian tube is one of the key structures responsible for the functional balance of the middle ear. Some clinical conditions associated with tubal malfunction can cause extremely unpleasant symptoms. These symptoms could be triggered by acute loss of weight, for example, after bariatric surgery.

Objective To evaluate the frequency and intensity of auditory tube dysfunction symptoms in obese patients after bariatric surgery.

Methods Nineteen patients with accepted formal indications for bariatric surgery underwent a hearing evaluation (otoscopy, tonal and vocal audiometry, and impedanceometry) and a hearing questionnaire before, at the time of, 3 months after surgery (first postoperative evaluation), and 6 months (second postoperative evaluation) after surgery. Patients with a history of ear disease or ear surgery were excluded.

Results None of the patients reported tubal dysfunction symptoms before surgery. Postsurgical results showed that 5 (26.3\%) patients presented symptoms related to dysfunction of the eustachian tube at the first postoperative evaluation. After the 6-

Keywords

- eustachian tube

- bariatric surgery

- weight loss month follow-up, 9 (47.3\%) patients reported symptoms of tubal dysfunction.

Conclusion This study suggests that bariatric surgery can cause symptoms of eustachian tube dysfunction, probably due to rapid weight loss and the consequent loss of peritubal fat.
\end{abstract}

\section{Introduction}

The eustachian tube, an osteocartilaginous canal that communicates the middle ear with the nasopharynx, is one of the most important structures responsible for the functional balance of middle ear structures and is involved in mechanisms of protection, aeration, and draining. ${ }^{1,2}$ Tubal malfunction can cause extremely unpleasant symptoms, such as autophony, cacophony, and the sensation of aural fullness. ${ }^{1,2}$ These symptoms might be unveiled by several clinical conditions, including the acute loss of weight after bariatric surgery. ${ }^{3}$

Bariatric surgery is already accepted as a treatment for morbid obesity and is becoming increasingly widespread

received

February 17, 2014

accepted

March 21, 2014

published online

July 18,2014
DOI http://dx.doi.org/

10.1055/s-0034-1382097. ISSN 1809-9777. among these patients. Morbid obesity is a multifactorial chronic disease and is genetically related to an excessive overstock of body fat. It is intimately related to medical, psychological, physical, and economic comorbidities. ${ }^{4}$ The obesity prevalence is rising among the world population, as is the number of surgeries. ${ }^{5}$ A 2009 Brazilian survey detected $14 \%$ obesity rate in Brazilian women, and in $4 \%$ of those, morbid obesity. Among men, $11 \%$ were obese and $2 \%$ morbidly obese. $^{5}$

Tube dysfunction after bariatric surgery might originate from the lack of fat tissue that involves and supports the eustachian tube. The abrupt decrease of adipocytes in this region caused by the acute loss of weight might lead to a
Copyright $\odot 2014$ by Thieme Publicações License terms Ltda, Rio de Janeiro, Brazil

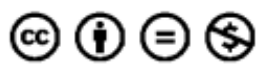


failure of tubal support and to a relaxation in this covering tissue, ultimately leading to eustachian tube dysfunction symptoms. Although symptoms of auditory alterations related to tube dysfunction are relatively frequent in these patients, only a few studies have investigated this relationship.

This study aims to evaluate the occurrence of signs and symptoms related to eustachian tube dysfunction in obese patients after bariatric surgery.

\section{Methods}

This prospective cohort study was conducted with adult patients ( $\geq 18$ years) with body mass index (BMI) $>40$ or $>35$ and presenting comorbidities related to their weight. All patients underwent bariatric surgery by production of gastric bypass (Fobi-Capella technique) at the same hospital, by the same surgeon. The hospital nutrition team in the postoperative follow-up evaluated all patients.

The institution's ethics committee approved this project and the data were collected between March and September 2011.

Prior to surgery, all patients had a hearing evaluation (otoscopy, tonal and vocal audiometry, and impedanceometry) and a standardized hearing questionnaire. There was no abnormality in those evaluations. Patients with disease or a history of ear surgery were excluded.

The questionnaire included questions about gender, age, current BMI, and symptoms related to tube dysfunction (aural fullness, sensation of water in ears, hearing own voice (echo), hearing crackles, tinnitus, hearing own breath, hearing own heartbeat).

Three to four months (first postoperative evaluation) and 5 to 6 months (second postoperative evaluation) after the surgery, all patients were reevaluated with the same questionnaire. Patients presenting with tubal dysfunction symptoms underwent a new hearing evaluation (otoscopy, tonal and vocal audiometry, and tympanometry).

\section{Results}

Twenty-one patients were selected for the study, but only 19 were included. Two were lost during follow-up. Thirteen $(68.4 \%)$ were female and six (31.6\%) were male. The age average and the overall median of BMI are shown at the - Table 1. No patients reported abnormal auditory symptoms before the bariatric surgery or had history of ear disease or surgery.

Three to four months after surgery (first postoperative evaluation), average weight loss among all patients was $31.57 \mathrm{~kg}$. Among women this average was $24.53 \mathrm{~kg}$ and among men, $32.25 \mathrm{~kg}$. After 5 to 6 months (second postoperative evaluation), the average loss of weight was $36.47 \mathrm{~kg}$. Among women this average was $33.15 \mathrm{~kg}$ and among men, $43.66 \mathrm{~kg}$. The values of BMI before surgery and at the first and second postoperative evaluation are shown in - Table 1.

The prevalence of tube dysfunction in the preoperative period was $0 \%$. In the postoperative evaluations, 5 (26.3\%) patients presented symptoms related to tube dysfunction at

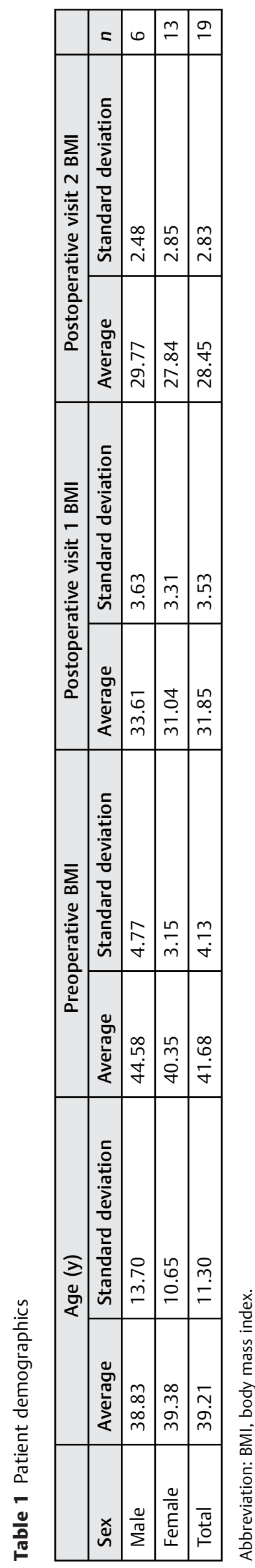




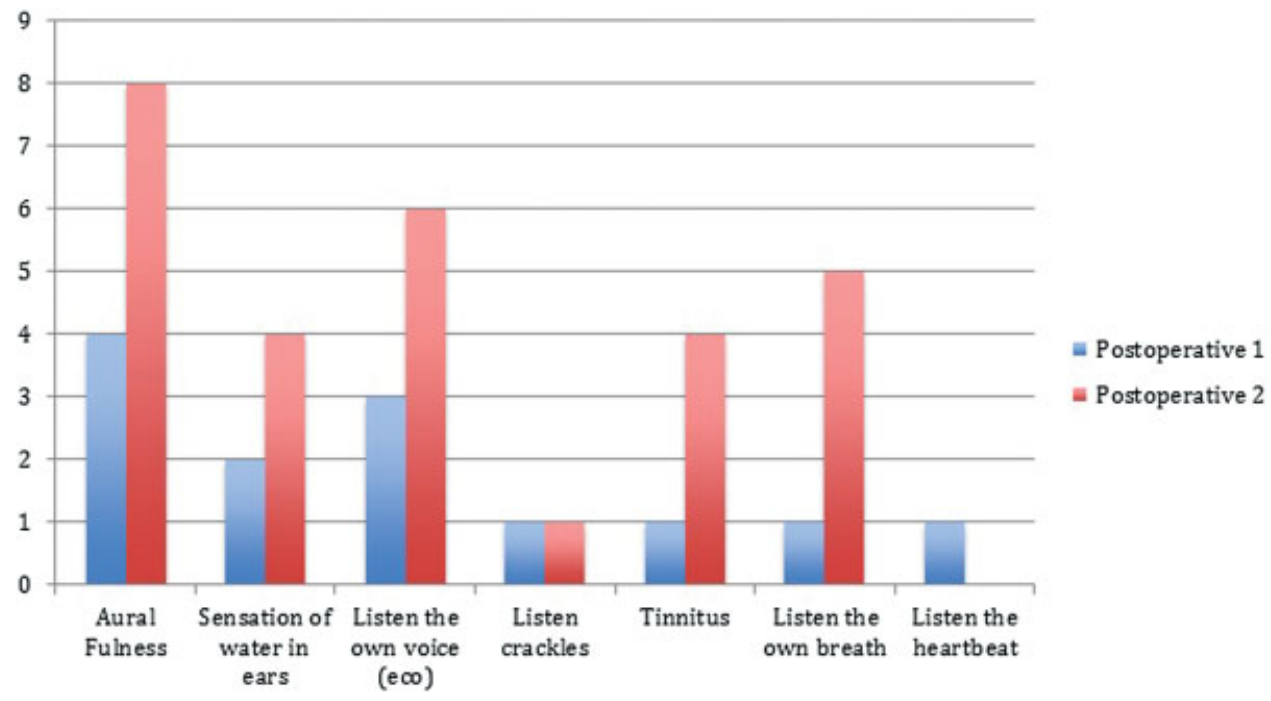

Fig. 1 Distribution of symptoms in postoperative visit 1 (3 to 4 months) and visit 2 ( 5 to 6 months), and number of patients who cited those symptoms.

the first evaluation, and 9 (47.3\%) presented symptoms at the second evaluation. Average onset of symptoms was 2.33 weeks (range: 1 to 20 weeks). The distribution of the most frequent symptoms can be seen in - Fig. 1.

Despite the frequency and intensity of the auditory complaints, no changes were detected after surgery with otoscopy and tympanometry.

\section{Discussion}

In an adult, the eustachian tube is usually $\sim 35$ to $38 \mathrm{~mm}$ in length, presenting $26 \mathrm{~mm}$ of fibrocartilaginous tissue. The lumen of this part of the tube is practically virtual, remaining closed due to its elastic properties, only opening at yawning, swallowing, or sneezing. ${ }^{1,2,6}$ The real mechanism of the tube's opening is still controversial, but it is believed that the tensor veli palatini, elevator veli palatine, and salpingopharyngeus muscles are involved. ${ }^{6}$

Factors involved in tubal dysfunction include loss of adipocyte tissue around the tube (Ostmann's fat), abnormal activity of peritubal muscles (tensor and elevator veli palatine and salpingopharyngeus), and inability of pterygoid venous plexus to assist the closure of the tube. ${ }^{3}$

Our study aimed to evaluate the relationship between acute loss of weight after bariatric surgery and the occurrence of tubal dysfunction symptoms. As the bariatric surgery is already ordained as treatment for morbid obesity and is becoming more and more widespread among this population, the impact in tubal function could be relevant.

All patients of this study had formal indications for surgery. Patients who lost weight faster were those with tubal dysfunction symptoms. Although the peak of weight loss occurred between 5 and 6 months, some auditory symptoms appeared 1 week after surgery, worsening with the progression of the loss of weight. It is interesting to note that despite the symptoms of tubal dysfunction, such as autophony, no patient presented alterations in audiometry and tympanometry. Unfortunately, objective diagnosis of tubal dysfunction is still difficult, as the symptoms are not always present at the examination time (e.g. tympanometry). The diagnosis remains based on the patient's history and clinical symptoms. $^{2,3}$

We believe that tubal dysfunction symptoms in our patients were related to the acute loss of adipocyte tissue that surrounds the cartilage part of the eustachian tube (Ostmann's fat). Other authors reported the same results in similar conditions of weight loss such as in restrictive alimentary diet, the puerperium period, and anorexia. ${ }^{1-3,7}$

Letti, in 1977, evaluated eight patients with complaints of patent tube; they all had a restrictive diet, with average weight loss of $15 \mathrm{~kg}$ in 45 to 65 days. The author observed that patients who submitted to very a restrictive diet with significant loss of weight or those with a severe disease, with poor general condition and nutrition, might present more permeability of the tube, predisposing its patency due to the loss of the peritubal adipocyte tissue. ${ }^{7}$

These studies strongly suggest that an acute and significant loss of weight can make these patients more susceptible to develop tubal dysfunction than the general population.

More studies are necessary to evaluate the relationship between loss of weight and occurrence of these symptoms and their intensity. A long-term follow-up with specific tests for tubal dysfunction would be useful to analyze the persistence and/or improvement of these symptoms and their relevance to the quality of life of these patients.

\section{Conclusions}

This study suggests that bariatric surgery can cause symptoms of eustachian tube dysfunction, probably due to rapid weight loss and the consequent loss of peritubular fat. 


\section{References}

1 Poe DS. Diagnosis and management of the patulous eustachian tube. Otol Neurotol 2007;28(5):668-677

2 Seibert JW, Danner CJ. Eustachian tube function and the middle ear. Otolaryngol Clin North Am 2006;39(6):1221-1235

3 Muñoz D, Aedo C, Der C. Patulous eustachian tube in bariatric surgery patients. Otolaryngol Head Neck Surg 2010;143(4):521-524

4 Ferraz EM, Arruda PCL, Bacelar TS, et al. Tratamento cirurgico da obesidade morbida. Rev Col Bras Cir 2003;30:98-105
5 Sociedade Brasileira de Cirurgia Bariatrica e Metabolica (SBCBM). Disponivel em: Available at: http://www.sbcb.org.br. Acessed in Oct 15th 2011.

6 Caldas SN, Mello JFJ, Martins RHG, Costa SS. Tratado de Otorrinolaringologia e Cirurgia Cervicofacial. 2nd ed. Chap. 14. São Paulo, Brazil: ROCA; 2011;14:260-261

7 Letti N. A tuba auditiva e o emagrecimento rapido. Rev Bras Otorrinolaringol 1977;43(2):160-164 